\title{
Emploi exclusif des concentrés protéiques de lactosérum dans la production de boissons fermentées
}

\author{
par \\ J. ROSSI* et L. COSTAMAGNA*
}

Les protéines du lactosérum, sélectivement purifiées par ultrafiltration des constituants en solution, non dénaturées, ayant intactes leurs propriétés fonctionnelles et biologiques, représentent une importante voie aux utilisations potentielles du lactosérum $[2,14,22,23,26$, 27], soit dans le cas d'un emploi exclusif $[6,28,29]$ que partiel $[1,6,10,11,17,24,25]$. En plus par leur composition en ce qui concerne les acides aminés, surtout les acides aminés essentiels [5], et l'équilibre parmi les acides aminés sulfurés, elles permettent une utile intégration avec des protéines d'origine végétale (mélanges sérumcéréales, sérum-légumes) [21].

Différents niveaux d'intégration lait-protéine du sérum ont été aussi utilement considérés dans la production de boissons fermentées lactiques $[4,19,24]$ de différents types microbiens, et lacto-alcooliques (Kefir) [4, 18].

Dans le même sens et après l'avoir déjà expérimenté pour l'obtention du Kefir [18], on a examiné dans cette note, l'emploi exclusif de protéines du sérum pour vérifier la production de boissons fermentées lactiques en même temps que la qualité protéique des produits obtenus.

\section{MATERIEL ET METHODES}

Préparation du concentré protéique du lactosérum et des boissons fermentées lactiques : la concentration du sérum a été obtenue

* Istituto di Microbiologia lattiero-casearia, Facoltà di Agraria di Perugia (Italia).

Etude effectuée avec le concours du Conseil National de la Recherche (CT 770028306 ) 
par ultrafiltration réalisée par une installation pilote munie d'une membrane Abcor type HFA-180, avec surface filtrante de $1 \mathrm{~m}^{2}$, dans les conditions suivantes : capacité $900 \mathrm{l} / \mathrm{h}$, pression à l'entrée 3,9 atm, pression à la sortie $1,8 \mathrm{~atm}$, température $50^{\circ} \mathrm{C}$. Le rétentat a été déshydraté par le système spray-Niro, ensuite il a été reconstitué à $10 \mathrm{p} .100(\mathrm{p} / \mathrm{v})$ dans l'eau et soumis à un traitement thermique $\left(80^{\circ} \mathrm{C} / 30 \mathrm{~min}\right)$ après avoir élevé sa valeur de $\mathrm{pH}$ à $6,9\left(5,8^{\circ} \mathrm{SH} \mathrm{p} .100\right)$.

A ce produit et comparativement au lait témoin, traité lui aussi thermiquement, on a inoculé (5 p. 100) une préculture en lait de $24 \mathrm{~h}$ de Lactobacillus acidophilus $\mathrm{P}$, Lactobacillus bifidus $\mathrm{RE}_{13}$, en souches isolées, et Lactobacillus bulgaricus $\mathrm{L}_{2}+$ Streptococcus thermophilus $\mathrm{S}_{2}$ en association. On a prolongé l'incubation à $40^{\circ} \mathrm{C}$ jusqu'à obtention du " coagulum ».

La caractérisation du lait, du concentré protéique et des boissons fermentées lactiques a été effectuée sur la base des paramètres suivants :

Evaluation chimique, physico-chimique et microbiologique : le contenu protéique a été mesuré selon Kjeldahl (Kjeltec System I-PBI) avec le facteur de conversion 6,38; le lactose selon la méthode AOAC [3], le $\mathrm{pH}$ par pH-mètre Metrohm Herisau E $300 \mathrm{~B}$, le titre d'acidité selon Soxelet-Henckel. On a effectué l'évaluation microbiologique sur APTG [16] pour L. acidophilus, L. bifidus, sur MRS [13] pour $L$. bulgaricus et APTs [16] pour Str. thermophilus.

Evaluation de la qualité protéique : on a limité l'examen de la composition en acides aminés aux boissons obtenues à partir du concentré protéique et du lait témoin après développement de $L$. bulgaricus et Str. thermophilus. Elle a été déterminée après hydrolyse acide ( $\mathrm{HCl} 6 \mathrm{~N}-120^{\circ} \mathrm{C}-24 \mathrm{~h}$ ) du produit lyophilisé, par la méthode chromatographique de Spackmann et al. [20]. On a déterminé le taux de tryptophane après l'hydrolyse alcaline avec l'hydroxyde de baryum [9]. La cystine et la méthionine ont été évaluées comme acide cystéique et méthionine-sulfone avec l'acide performique et l'hydrolyse acide [15].

La digestibilité in vitro (EUD*), c'est-à-dire l'indice des acides aminés du digestat enzymatique ultrafiltré, a été calculé selon la méthode de Floridi et Fidanza [8] en dosant dans l'ultrafiltrat les aminoacides rendus disponibles, après digestion dans une cellule de UF Amicon, de l'échantillon avec pepsine, trypsine, pancréatine et érepsine.

\section{RESULTATS}

Les produits obtenus, qui sont assimilés à des produits de nouvelle formulation, ont été examinés au terme de la phase de «coagu-

* Enzymatic Ultrafiltrate Digest (Aminoacid index). 
lation ". Ils sont caractérisés par une consistance notable, supérieure à celle du lait témoin. Leur structure est homogène, non plastique, résistante à la coupe, qui est nette, avec une surface de rupture luisante.

L'aspect organoleptique répète celui des produits d'origine. D'autres données, telles que l'aspect chimique et microbiologique, démontrent aussi que l'emploi des protéines du sérum convient pour la préparation des boissons fermentées. L'observation relative au premier aspect conduit à relever un contenu protéique égal à 5,92 p. 100 (tab. 1)

\section{TABLEAU 1}

Composition chimique et physico-chimique du concentré protéique, reconstitué à 10 p. 100 et du lait témoin

\begin{tabular}{l|c|c}
\hline & Concentré protéique & Lait témoin \\
\hline pH & 6,90 & 6,65 \\
& 5,80 & 7,50 \\
SH p. 100 & 0,51 & 4,30 \\
Lactose (g p. 100) & 5,92 & 2,67 \\
\hline
\end{tabular}

avec des taux de lactose résiduel dans les boissons compris entre 0,36 et 0,62 p. 100 (tab. 2). Il est possible que le taux plus élevé, qui surpasse même le taux initial, soit dû à la concentration effectuée dans la phase d'incubation qui, pour $L$. acidophilus et $L$. bifidus a été supérieure à $20 \mathrm{~h}$.

A quelques exceptions près, la situation microbiologique peut aussi être interprétée favorablement. Le profil des micro-organismes présents après coagulation (tab. 2) ne montre pas de différences par rapport aux boissons obtenues à partir du lait dans le cas où sont impliqués $L$. bifidus et $L$. bulgaricus tandis que le contrôle de $L$. acidophilus et Str. thermophilus, en association avec L. bulgaricus, révèle un aspect légèrement différent.

La qualité protéique des boissons, contrôlée après le développement de L. bulgaricus en association avec Str. thermophilus, a été considérée en fonction du profil des aminoacides et de la digestion enzymatique in vitro (EUD) (tab. 3) dans le cas de l'emploi du concentré protéique et du lait témoin.

Le critère des acides aminés se définit en faveur des boissons sériques, en particulier en ce qui concerne les aminoacides essentiels. 


\section{TABLEAU 2}

Composition chimique, physico-chimique et microbiologique des boissons obtenues avec le concentré protéique du sérum et le lait témoin

\begin{tabular}{|c|c|c|c|c|c|c|}
\hline Type de boisson & $\mathrm{pH}$ & $\begin{array}{l}{ }^{\circ} \mathrm{SH} \\
\text { p. } 100\end{array}$ & $\begin{array}{l}\text { Lactose } \\
\text { g p. } 100\end{array}$ & $\begin{array}{c}\text { Lactobacilles } \\
\mathrm{N} / \mathrm{g}\end{array}$ & $\begin{array}{c}\text { Streptocoques } \\
\mathrm{N} / \mathrm{g}\end{array}$ & $\begin{array}{c}\text { Temps de } \\
\text { coagulation } \\
\text { (h) }\end{array}$ \\
\hline \multicolumn{7}{|l|}{ Concentré protéique : } \\
\hline L. acidophilus $\mathrm{P}$ & 5,65 & 13,75 & 0,62 & $50 \times 10^{5}$ & & 21 \\
\hline L. bifidus $\mathrm{RE}_{13}$ & 5,50 & 16,75 & 0,55 & $32 \times 10^{7}$ & & 24 \\
\hline L. bulgaricus $\mathrm{L}_{2}$ et Str. thermophilus $\mathrm{S}_{2}$ & 4,80 & 21,50 & 0,36 & $49 \times 10^{4}$ & $84 \times 10^{6}$ & 4,30 \\
\hline \multicolumn{7}{|l|}{ Lait témoin : } \\
\hline L. acidophilus $\mathrm{P}$ & 5,20 & 24,25 & 4,15 & $46 \times 10^{7}$ & & 21 \\
\hline L. bifidus $\mathrm{RE}_{13}$ & 5,30 & 21,00 & 4,15 & $37 \times 10^{7}$ & & 24 \\
\hline L. bulgaricus $\mathrm{L}_{2}$ et Str. thermophilus $\mathrm{S}_{2}$ & 4,30 & 34,25 & 3,85 & $16 \times 10^{4}$ & $44 \times 10^{7}$ & 6,45 \\
\hline
\end{tabular}


TABLEAU 3

Evaluation de la qualité protéique (aminoacides totaux g/16 g N-EUD) des boissons fermentées obtenues à partir du concentré protéique du sérum et du lait témoin

\begin{tabular}{|c|c|c|c|c|c|c|c|c|c|}
\hline & \multicolumn{2}{|c|}{ Aminoacides totaux* } & \multirow{2}{*}{$\begin{array}{c}\text { Profil } \\
\text { FAO/WHO } \\
\text { (7) }\end{array}$} & \multicolumn{2}{|c|}{$\Delta$ p. $100^{* *}$} & \multicolumn{2}{|c|}{$\begin{array}{c}\text { Aminoacides après } \\
\text { digestion enzymatique }\end{array}$} & \multicolumn{2}{|c|}{$\begin{array}{c}\text { Aminoacides libres } \\
\text { (p. 100) }\end{array}$} \\
\hline & $\begin{array}{l}\text { Concentré } \\
\text { protéique }\end{array}$ & $\begin{array}{l}\text { Lait } \\
\text { témoin }\end{array}$ & & $\begin{array}{l}\text { Concentré } \\
\text { protéique }\end{array}$ & $\begin{array}{l}\text { Lait } \\
\text { témoin }\end{array}$ & $\begin{array}{l}\text { Concentré } \\
\text { protéique }\end{array}$ & $\begin{array}{l}\text { Lait } \\
\text { témoin }\end{array}$ & $\begin{array}{l}\text { Concentré } \\
\text { protéique }\end{array}$ & $\begin{array}{l}\text { Lait } \\
\text { témoin }\end{array}$ \\
\hline Ac. aspartique & 10,34 & 7,32 & & & & 1,12 & 0,74 & 11 & 10 \\
\hline Thréonine & 6,69 & 3,55 & 4,0 & $+67,25$ & $-11,25$ & 1,30 & 0,66 & 19 & 19 \\
\hline Sérine & 4,18 & 4,74 & & & & 0,56 & 0,45 & 13 & 9 \\
\hline Ac. glutamique & 23,80 & 24,91 & & & & 2,92 & 2,44 & 12 & 10 \\
\hline Proline & 4,70 & 7,26 & & & & 0,20 & 0,30 & 4 & 4 \\
\hline Glycine & 2,07 & 2,05 & & & & 0,60 & 0,55 & 29 & 27 \\
\hline Alanine & 5,96 & 3,48 & & & & 1,43 & 0,98 & 24 & 28 \\
\hline Valine & 6,98 & 6,50 & 5,0 & $+39,60$ & $+30,00$ & 2,42 & 0,80 & 35 & 12 \\
\hline Cystine & 2,11 & 0,847 & 35 & -1428 & -1885 & 0,09 & 0,10 & 4 & 12 \\
\hline Méthionine & 0,89 & $2,00 \mathrm{~J}$ & 3,5 & $-14,28$ & $-18,85$ & 0,41 & 0,47 & 46 & 23 \\
\hline Isoleucine & 8,10 & 5,47 & 4,0 & $+102,50$ & $+36,75$ & 1,47 & 0,94 & 18 & 15 \\
\hline Leucine & 12,48 & 9,82 & 7,0 & $+78,28$ & $+40,28$ & 5,04 & 3,68 & 40 & 37 \\
\hline Tyrosine & 3,34 & $3,57]$ & 60 & +2133 & +4100 & 2,42 & 1,92 & 72 & 54 \\
\hline Phénylalanine & 3,94 & $4,89 \mathrm{~J}$ & 6,0 & $+21,33$ & $+41,00$ & 2,17 & 1,81 & 55 & 37 \\
\hline Lysine & 9,96 & 8,42 & 5,5 & $+81,09$ & $+56,36$ & 5,47 & 3,17 & 55 & 38 \\
\hline Histidine & 1,66 & 2,64 & & & & 0,41 & 0,44 & 25 & 17 \\
\hline Arginine & 2,42 & 3,08 & & & & 2,02 & 1,61 & 83 & 52 \\
\hline Tryptophane & 1,71 & 1,32 & 1,0 & $+71,00$ & $+32,00$ & 0,86 & 0,63 & 50 & 48 \\
\hline $\mathrm{N}$ total (p. $100 \mathrm{ss})$ & 11,30 & 6,05 & & & & & & & \\
\hline Indice EUD & 81,78 & 73,94 & & & & & & & \\
\hline
\end{tabular}

Ac. aspartique

Ac. glutamique

Proline

Alanine

Méthionine

Isoleucine

Leucine

Phénylalanin

Arginine

* Valeur moyenne des deux déterminations après deux hydrolyses différentes.

** Pourcentage d'écart par rapport au modèle FAO/WHO. 
Parmi ceux-ci, thréonine, isoleucine, leucine et lysine représentent les constituants majeurs alors que méthionine et phénylalanine, dans la mesure où elles sont limitées constitutivement dans les protéines du sérum [12], restent également à un bas niveau.

Des indications ultérieures d'évaluation peuvent résulter de la comparaison avec le profil proposé provisoirement par le groupe FAO/WHO [7] (tab. 3).

On constate généralement une élévation significative de la disponibilité des acides aminés essentiels dans la boisson obtenue avec le concentré protéique. La déficience en thréonine du yoghourt test $(-11,25$ p. 100) est largement compensée $(+67,25$ p. 100) tandis que les autres carences (cystine + méthionine) persistent dans les deux cas.

Le taux des acides aminés libérés après la digestion enzymatique met en évidence la meilleure qualité biologique de la boisson produite avec le concentré protéique. A l'exception de l'alanine et de la cystine, les niveaux de disponibilité potentielle sont généralement plus élevés et s'expriment par des valeurs notables pour la valine (35 contre 12), méthionine (46 contre 23 ), tyrosine (46 contre 37), lysine (55 contre 38 ), arginine (83 contre 52 ).

Selon cette évaluation aussi, ce sont surtout les acides aminés essentiels qui sont intéressés, fournissant au point de vue nutritionnel, une réponse favorable qui se traduit par une valeur de EUD égale à 81,78 contre 73,94 pour la boisson témoin.

\section{Remerciements}

Nous adressons nos remerciements à M. le $\operatorname{Pr}$ F. Fidanza, Directeur de l'Institut des Sciences de l'Alimentation de la Faculté de Pharmacie pour avoir contribué à cette étude en relevant la valeur nutritionnelle des boissons fermentées lactiques.

\section{Résumé}

On a considéré l'emploi exclusif des protéines du lactosérum, concentrées par ultrafiltration, dans la production de boissons fermentées de type microbien différent : L. acidophilus, L. bifidus, en culture isolée, L. bulgaricus et Str. thermophilus, en association.

Le concentré protéique, déshydraté par le système spray, a été reconstitué à $10 \mathrm{p}$. 100 et traité thermiquement $\left(80^{\circ} \mathrm{C} / 30 \mathrm{~min}\right)$ après avoir modifié sa valeur de $\mathrm{pH}(6,9)$.

Les concentrés protéiques, traités ensuite selon le processus d'obtention des boissons fermentées lactiques, ont donné, en comparaison avec le lait témoin, un produit de structure homogène plus compacte, assimilable organoleptiquement aux produits d'origine, avec 
un contenu protéique plus élevé $(5,92 \mathrm{p} .100)$ et des valeurs de lactose résiduel très limitées $(0,36-0,62 \mathrm{p}$. 100). La situation microbiologique elle-même peut être, avec des exceptions limitées, interprétée favorablement.

La qualité protéique de la boisson, notée seulement en présence de l'association $L$. bulgaricus - Str. thermophilus, confirme la validité des concentrés protéiques par rapport au lait.

Le profil aminoacide, avec référence particulière au taux des acides aminés essentiels, et leur plus grande disponibilité après le processus de digestion enzymatique, garantissent aux concentrés protéiques une qualité biologique élevée qui se traduit par une valeur de EUD égale à 81,78 contre 73,94 pour la boisson témoin.

\section{S u m m a ry}

\section{LACTIC ACID BEVERAGES OBTAINED BY WHEY PROTEIN CONCENTRATES ALONE}

Ultrafiltered whey proteins were employed alone to produce lactic acid beverages with different types of microorganisms: L. acidophilus, L. bifidus, as isolated cultures, L. bulgaricus and Str. thermophilus in association.

The proteic concentrate was spray-dried, reconstituted at 10 p. 100, and after modifiyng its $\mathrm{pH}$ value at 6,9 , treated at $80^{\circ} \mathrm{C} / 30 \mathrm{~min}$.

The organoleptic sensory evaluation of the beverages, obtained following the normal processus, reproduce the original products; their structure was homogenously firm; protein was 5,92 p. 100, lactose between $0,36-0,62$ p. 100 . Also the microbiological evaluation was generally favorable and like that of reference milk beverage.

Nutritional value of whey protein beverages was pointed out in the presence of L. bulgaricus and Str. thermophilus. The pattern of essential aminoacids and their availability after enzymatic digestion, assure high biological value, as confirmed also by EUD value of 81,87 against 73,94 of the milk test beverage.

Reçu pour publication en novembre 1980.

\section{Bibliographie}

[1] Abrahamsen (R. K.) (1979). - Cheesemaking from milk fortified with ultrafiltrated whey protein concentrate. Milchwissenschaft, 34, 65.

[2] Adrian (J.) (1971). - A propos des poudres de lait et des lactoprotéines levurées. Ind. Alim. Agric., 88, 1607.

[3] A.O.A.C. (1970). - Official methods of analysis of the Association of Official Analytical Chemist. Washington, D.C. 
[4] Chojnowski (W.), Poznanski (S.), Smietana (Z.) et Bednarski (W.) (1978). Application de l'ultrafiltration dans la fabrication des boissons fermentées à partir du lait et du sérum. XX Int. Dairy Congr., brèves comm., Paris, 961.

[5] Christensen (V.W.) (1975). - Fourth Int. Marschall Dairy Symposium London.

[6] Emaldi (G.C.) (1975). - Recupero delle siero-proteine del formaggio. Ind. latte, 11, 89.

[7] FAO/WHO (1973), - Energy and protein requirements.

[8] Floridi (A.) e Fidanza (F.) (1975). - Sulla qualità delle proteine alimentari. Nota III. Indice aminoacidico sul digerito enzimatico ultrafiltrato (EUD). S.e TA-NU, 5, 1.

[9] Knox (T.), Kohler (G. O.), Palter (R.) and Walker (H. G.) (1980). Anal. Biochem., 36, 136.

[10] Krasheninin (P. F.), Gorelova (N. F.), Silaeva (V. M.), Sakharov (S. D.), TOPURidZE (A. D.) and Kulikov (V. S.) (1978). - Effect of denatured whey proteins on the quality of soft Shkol'nyi cheese. Trudy Vsesoyuznyi Nauchno-issledovatel'skii Institut Maslodel'noi Syrodel'noi Promyshlennosti, 23, 89.

[11] Krasheninin (P. F.) and Nebert (V. K.) (1975). - Study of possibility of manufacturing hard rennet cheeses with addition of whey proteins tomilk. Trudy, Vsesoyuznyi Nauchno-issledovatel'skii Institut Maslodel'noi i Syrodel'noi Promyshlennosti, 18, 27.

[12] Ling (E. R.), Kon (S. K.) and Porter (J. W. G.) (1961). - In Milk: The mammary gland and its secretion. Acad. Press, 2, 210.

[13] Man (J. C. DE) Rogosa (M.) and Sharpe (M. E.) (1960). - A Medium for the cultivation of lactobacilli. J. Appl. Bacteriol., 23, 130.

[14] Mc Donough (F. E.), Hargrove (R. E.), Mattinly (W. W.), Posati (L. P.) and ALFORD (J. A.) (1974). - Composition and properties of whey protein concentrates from ultrafiltration. J. Dairy Sci., 57, 1438.

[15] Moore (S.) (1963). - On the determination of cystine as cysteic acid. J. Biol. Chem, 238, 235.

[16] Raibaud (P.), Caulet (M.), Galpin (J.,W) et Mocouot (G.). (1961). J. Appl. Bacteriol,, 24, 285.

[17] Ramazanov (I. V.) and Pankova (M. S.) (1978). - Whey proteins and their use in cheesemaking. Molochnaya Promyshlennost, 5, 8.

[18] Rossi (J.) e Rossi (J.) (1980). - Le proteine del siero recuperate per ultrafiltrazione nell'ottenimento del kefir e come mezzo per valutare la riproduzione dei granuli. Sci. Tecn. Latt. Cas., 31, 241.

[19] Rossi (J.) e Costamagna (L.). - Integrazione del latte con siero ultrafiltrato nella produzione di bevande lattiche. Ann. Fac. Agr. Perugia, in press di stampa.

[20] Spackmann (D. H.), Stein (W. H.) and Moore (S.) (1958). - Chromatography of Aminoacids on Sulfonated Polystyrene Resins. An improved System. Anal. Chem., 30, 1185.

[21] Spadoni (M. A.) (1978). - - Il valore nutritivo dei costituenti del siero di caseificazione. Sci. Tecn. Latt. Cas., 29, 344.

[22] Suter (R.) and Puhan (Z.) (1977). - Some technologically important functional properties of whey protein. Deutsche Molkerei-Zeitung, 98, 349.

[23] Suter (R.) and WanNeR (E.) (1977). - Some technologically important functional properties. Whippability and gelling capacity of whey protein concentrates. Deutsche Molkerei-Zeitung, 98, 532. 
[24] Toppino (P.) (1978). - Utilizzazione delle proteine del siero di latte nella preparazione dei prodotti lattiero-caseari. CNR. Recupero et utilizzazione delle proteine del siero di latte. Progetto finalizzato «Ricerca di nuove fonti proteiche e nuove formulazioni alimentari». Subprogetto n. 4. Ed. Tecnos S; p.a., Milano.

[25] Tsvetkova (N. D.), Lebedeva (G. V.), Ramazanov (I. V.), Kudryashova (M. M.) and Konovalova (T. M.) (1975. - Use of dried concentrated cheese whey in processed cheese manufacture. Trudy. Vsesoyuznyi Nauchno-issledovatel'skii Institut Maslodel'noi i Syrodel'noi Promyshlennosti, 20, 74.

[26] Wingered (W. H.) (1971). - Lactalbumin as a food ingredient. J. Dairy Sci., $54,1234$.

[27] Wingered (W. H.), Shperstein (C.) and Lutwak (L.) (1970). - Bland, soluble whey protein concentrate as excellent nutritional properties. Food Technol., 24, 34.

[28] Zerfurides (G. K.) and ManolKides (K. Z.) (1977). - Standardization of a method of curd production from cows'milk Teleme cheese whey. Geoponika, 23, 125.

[29] Zerfurides (G. K.) and Manolkides (K. Z.) (1978). - Study on a new type of whey cheese. Milchwissenschaft, 33, 617. 